\title{
L’intégration économique continentale et ses effets sur les gouvernements infra-étatiques : de l'ALE à l'ALENA et au-delà
}

\section{Dorval Brunelle, Yves Bélanger et Christian Deblock}

Numéro 32, 1999

La politique en otage

URI : https://id.erudit.org/iderudit/1002400ar

DOI : https://doi.org/10.7202/1002400ar

Aller au sommaire du numéro

\section{Éditeur(s)}

Département de sociologie - Université du Québec à Montréal

ISSN

0831-1048 (imprimé)

1923-5771 (numérique)

Découvrir la revue

Citer cet article

Brunelle, D., Bélanger, Y. \& Deblock, C. (1999). L’intégration économique continentale et ses effets sur les gouvernements infra-étatiques : de l'ALE à l'ALENA et au-delà. Cahiers de recherche sociologique, (32), 85-117. https://doi.org/10.7202/1002400ar
Résumé de l'article

Par le passé, les accords de libre-échange et autres ententes commerciales engageaient au premier chef leurs signataires; ils n'avaient pas d'effets directs sur les autres niveaux de gouvernement. Or cette étanchéité entre les niveaux de gouvernement ne prévaut plus depuis l'entrée en vigueur, le 1er janvier 1989, de l'Accord de libre-échange entre le Canada et les États-Unis. En effet, pour la première fois, un accord commercial stipule expressément que ses dispositions devraient s'appliquer également aux États et aux provinces. La question surgit alors de savoir en quoi et comment l'ALE, puis l'Accord de libre-échange nord-américain, en 1994, sont venus modifier les relations et interrelations entre les pouvoirs centraux et les gouvernements infra-étatiques. L'article se propose d'aborder ces questions dans une perspective d'ensemble tenant compte des trois Amériques. L'analyse est partagée en trois temps. Dans un premier temps, les auteurs présentent quelques données de base sur les pays et les gouvernements infra-étatiques. Dans un deuxième temps, ils examinent les dispositions pertinentes de l'ALE et de l'ALENA, après quoi ils s'intéressent aux pouvoirs provinciaux ou étatiques en tant qu'acteurs politiques. Cette approche leur permet de mettre en lumière quelques nouveaux aspects de la démocratie d'exécutifs qui semble prédominer dans la mise en place des cadres normatifs propres à la mondialisation économique dans sa phase actuelle. 


\section{L'intégration économique continentale et ses effets sur les gouvernements infra- étatiques: de l'ALE à l'ALENA et au-delà}

Dorval BRUNELLE, Yves BÉLANGER et Christian DEBLOCK

Nous voudrions, dans les pages qui suivent, fournir des éléments de réponse à la question de savoir en quoi et comment les formes actuelles de régionalisme économique ou, plus généralement, d'intégration économique continentale, sont susceptibles d'avoir des effets sur les niveaux inférieurs ou infra-étatiques de gouvernement et, plus spécialement, sur les relations et interrelations entre les niveaux de gouvernement eux-mêmes, d'une part, et sur les rapports entre gouvernements et sociétés, d'autre part. Nous cherchons à voir dans quelle mesure l'intégration économique à grande échelle favoriserait à la fois la démocratisation du pouvoir politique et l'imputabilité de ceux qui l'exercent. À la vérité, il semble que ce ne soit pas le cas, et certains indices peuvent servir à préciser la nature du problème. Nous en retiendrons deux pour éclairer notre propos. Le premier indice, c'est l'accroissement de la confidentialité dans le traitement des dossiers qui, à leur tour, couvrent un nombre croissant de domaines et d'enjeux; c'est sans doute un des paradoxes les plus troublants de cet après-guerre froide que de nous trouver face à la démultiplication du recours au secret de la part des acteurs engagés dans des négociations et autres tractations commerciales, en particulier, alors même que les questions traitées gagnent en ampleur et en conséquence ${ }^{1}$. L'exemple qui vient à l'esprit, c'est, bien sûr, celui des négociations concernant la libéralisation des échanges et autres accords sur l'investissement, où la confidentialité demeure une exigence forte pour les négociateurs, tandis que la nature des questions traitées a depuis longtemps quitté le

1 Voir D. P. Moynihan, Secrecy: The American Experience, cité dans G. Wills, «Bully of the free world», Foreign Affairs, mars-avril 1999, p. 55. Wills relève que le nombre de secrets s'est considérablement accru depuis la fin de la guerre froide, avec une augmentation de $62 \%$ de documents dont la divulgation éventuelle constituerait une menace pour la sécurité nationale des États-Unis. 
domaine du commerce au sens propre et couvre désormais des questions sociales, syndicales, environnementales et autres. Le second indice, quant à lui, nous renvoie à la constance dans les politiques suivies et assumées par les gouvernements au-delà des changements qui se produisent dans la gouverne politique elle-même. Toujours dans le domaine du libre-échange, tel premier ministre ou tel président qui s'était affiché en adversaire d'accords de libéralisation du commerce avant d'être élu aura tôt fait de changer son fusil d'épaule une fois parvenu au pouvoir. On peut sans doute imputer au cynisme les revirements que nous ont servis le président Bill Clinton ou le premier ministre Jean Chrétien qui s'étaient tous deux engagés à dénoncer l'Accord de libre-échange nord-américain (ALENA) alors qu'il était en cours de négociation, ou imputer à l'opportunisme la décision prise par le gouvernement péquiste quand il a intégré l'ALENA à sa légistation, à l'instar du gouvernement de l'Alberta et contrairement à ce que fera celui de l'Ontario, il reste qu'il s'agit là d'explications superficielles qui ne vont pas au-delà des apparences. Car, dans tous ces cas, il convient de mettre en lumière un phénomène beaucoup plus troublant au fond qui est celui de l'imputabilité des détenteurs de pouvoir, en particulier du pouvoir exécutif, qui semblent agir à l'intérieur d'un réseau de contraintes sur lesquelles les citoyens n'ont plus de prise. L'imputabilité apparaît aujourd'hui comme une contrainte qui s'exerce de l'extérieur sur des élites politiques domestiques qui, en se pliant à de semblables exigences, privent, du coup, leurs commettants de leurs propres prérogatives et, parmi celles-ci, d'un contrôle minimal sur les mandats qui sont remplis et exercés en leurs nom et place.

C'est donc l'ampleur même des questions envisagées et traitées sous le couvert des accords de libre-échange qui nous conduit à étudier leurs effets sur les relations et interrelations entre niveaux de gouvernement, et, pour ce faire, nous allons centrer notre attention sur les niveaux infra-étatiques qui se trouvent placés au cœur des engagements souscrits par les pouvoirs centraux à l'heure actuelle. Par là, nous désignons, de manière générale, non seulement les unités formant partie des fédérations que l'on désigne soit comme des États aux États-Unis, au Mexique, au Venezuela et au Brésil, soit comme des provinces en Argentine et au Canada, mais également les gouvernements locaux que l'on assimile souvent aux seuls pouvoirs municipaux. Si l'expression "gouvernement infra-étatique» nous semble préférable à celle de gouvernement non souverain ${ }^{2}$, à celle de gouvernement non central ${ }^{3}$ ou

2 A. O. Hero et L. Balthazar, Contemporary Québec and the United States: 1960-1985, Cambridge, University Press of America, 1988.

3 E. H. Fry, «North American municipalities and their involvement in the global economy», dans P. K. Kresl et G. Gappert (dir.), North American Cities and the Global 
à celle de gouvernement sous-national ${ }^{4}$, c'est pour la simple raison que, d'une part, cette qualification apparaît plus neutre que les autres et que, d'autre part, elle met d'entrée de jeu en lumière le fait que l'État comprend ou englobe toujours plusieurs niveaux, central, intermédiaire et local, de gouvernement. Or il arrive encore trop souvent, sous l'influence de théories politiques fortement marquées par un républicanisme d'inspiration jacobine notamment, que l'on ait recours à la notion d'État pour désigner le seul niveau central de gouvernement, alors que, dans des régimes fédéralistes, en particulier, nous avons affaire à deux niveaux qui sont souverains dans leurs champs de compétence propres, ce que reflète au mieux le fait que ce soit les niveaux intermédiaires qui aient été désignés comme des États à l'intérieur de plusieurs fédérations. Par ailleurs, le recours au qualificatif national fait l'objet d'une double revendication, à la fois de la part d'un gouvernement provincial et de la part du gouvernement fédéral dans le contexte canadien, et c'est ce qui explique que nous ayons préféré nous éloigner de la désignation de gouvernement «sous-national». $\mathrm{Au}$ demeurant, ce flottement dans les qualifications reflète sans doute un problème plus fondamental qui est celui de savoir s'il existe bel et bien un statut constitutionnel équivalent d'une fédération à l'autre entre les niveaux de gouvernement dans les Amériques au-delà du fait que les constitutions des pays en question instaurent des régimes fédéraux fortement inspirés du modèle américain.

Dans le même ordre d'idée, non seulement les six fédérations des Amériques intègrent-elles deux niveaux plus ou moins étanches de gouvernement dits «souverains» dans leurs aires de compétence propres, mais elles comptent également, nous venons de le noter, des gouvernements locaux, voire des gouvernements urbains, qui diffèrent les uns des autres par la taille, bien sûr, mais aussi par leur statut juridique. Quoi qu'il en soit de cette prolifération des lieux de pouvoir et de gestion, que l'on envisage l'intégration économique sous un angle spatial, sous un angle institutionnel ou sous un angle normatif, ce qui nous préoccupera, ce sera la prise en compte des effets du régionalisme économique sur l'articulation entre ces différents niveaux de gouvernement.

Ce n'est d'ailleurs pas par hasard que ce genre de questionnement

Economy. Challenges and Opportunities, Thousand Oaks (Calif.), «Urbain Affairs Annual Review», 44, 1995.

4 P. Soldatos, «La Communauté européenne et la zone de libre-échange canadoaméricaine: quelques niveaux de comparaison de leurs processus intégratifs», dans D. Brunelle et C. Deblock (dir.), L'Amérique du Nord et l'Europe communautaire: intégration économique, intégration sociale?, Sainte-Foy, Presses de l'Université du Québec, p. 77-96. 
fait surface dans la littérature spécialisée depuis quelque temps. En effet, autant, par le passé, les dimensions externe et interne de l'intégration économique étaient maintenues dans des aires relativement étanches, avec le résultat que les engagements conclus entre pays souverains avaient peu d'effets juridiques ou constitutionnels directs sur les autres niveaux de gouvernement, autant, depuis peu, les nouvelles formes et modalités de l'intégration engagent également les différents niveaux de gouvernement et bousculent, dans certains cas, les relations et interrelations qui existaient entre eux jusque-là. Un exemple récent et révélateur de cette nouvelle approche nous est fourni par la diffusion, à l'automne 1997, d'une version préliminaire de l'Accord multilatéral sur l'investissement (AMI), qui était alors en cours de négociation au sein de l'Organisation de coopération et de développement économiques (OCDE), qui prévoyait expressément que ses dispositions engageraient non seulement les gouvernements centraux, mais également les gouvernements «infra-nationaux» de même que les gouvernements locaux. Or, malgré ce qu'en ont dit plusieurs analystes et critiques, il ne s'agissait pas là d'une innovation ni d'une singularité, puisqu'on trouvait déjà des dispositions analogues dans l'Accord de libre-échange entre le Canada et les États-Unis de 1989 (ALE, art. 103) et dans l'Accord de libre-échange nord-américain de 1994 (ALENA, art. 105), qui constituent tous deux, sur ces questions, les précédents les plus significatifs qui se puissent rencontrer.

Nous aurons bientôt l'occasion de revenir sur l'exemple canadien à ce propos, essentiellement parce que nous disposons, dans ce cas-là, d'un document d'une pertinence certaine, le Rapport de la Commission royale sur l'union économique et les perspectives de développement du Canada ${ }^{5}$, mieux connu sous le nom de rapport Macdonald. Non seulement ce rapport a-t-il engagé une critique de fond de l'économie politique d'inspiration keynésienne qui avait servi de cadre général aux interventions publiques dans l'économie et la société au cours des quatre décennies précédentes, une critique qui légitime ce réalignement théorique et programmatique majeur qui conduit les commissaires à faire du libre-échangisme et de la signature d'un éventuel accord de libre-échange avec les États-Unis leur recommandation centrale ${ }^{6}$, mais il

5 Rapport de la Commission royale sur l'union économique et les perspectives de développement du Canada, Ottawa, ministère des Approvisionnements, 1985, 3 vol. et annexe.

6 Y. Bélanger et D. Brunelle (dir.), L'ère des libéraux. Le pouvoir fédéral de 1963 à 1984, Québec, Presses de l'Université du Québec, 1988 ; C. Deblock et D. Brunelle, «Le régionalisme économique international: de la première à la deuxième génération», dans M. Fortmann, S. N. Macfarlane et S. Roussel (dir.), Tous pour un ou chacun pour soi. Promesses et limites de la coopération régionale en matière de sécurité, Québec, Institut québécois des hautes études internationales, 1996, p. 271-315; D. Brunelle, Droit et exclusion. Critique de l'ordre libéral, Montréal et Paris, L'Harmattan, 1997. 
propose également une révision significative des relations et interrelations entre les trois paliers de gouvernement, les pouvoirs fédéral, provincial et municipal. Nous disposons ainsi, avec le rapport Macdonald, d'une étude qui a poussé le souci de cohérence jusqu'à lier le recours aux paramètres du néolibéralisme à la réarticulation des pouvoirs et responsabilités entre les différents paliers de gouvernement, et c'est ce qui en fait une pièce majeure à verser au dossier des effets de l'intégration sur les niveaux de gouvernement.

L'analyse comprend trois parties: la première fournit quelques données de base sur les gouvernements dans les Amériques afin de donner une idée générale de l'ampleur de la question étudiée, puis elle reprend quelques-uns des arguments les plus pertinents de la commission Macdonald sur le rapport entre le libre-échange et les modifications constitutionnelles suggérées afin de réaménager les relations entre les niveaux de pouvoir; la deuxième partie fait un survol des dispositions pertinentes de l'ALE et de l'ALENA afin de mettre en lumière leurs effets sur les pouvoirs infra-étatiques; la troisième partie, enfin, est consacrée à la prise en compte du rôle des unités infraétatiques en tant qu'acteurs politiques dans le contexte actuel de la mondialisation des économies et de la mise en place d'une Zone de libre-échange des Amériques (ZLEA).

\section{Description de l'unité d'analyse et problématique}

\subsection{Quelques données}

L'Amérique du Nord comprend trois fédérations, le Mexique, les États-Unis et le Canada, à l'intérieur desquelles les fonctions de l'État sont partagées entre trois niveaux de gouvernement dont deux bénéficient, sur le plan constitutionnel, de la souveraineté législative dans leurs aires de compétence respectives, les gouvernements fédéraux et les gouvernements provinciaux ou étatiques, tandis que le troisième palier, qui n'a pas d'autonomie constitutionnelle propre, tombe sous l'autorité des gouvernements provinciaux ou étatiques. $\mathrm{Au}$ niveau intermédiaire, on dénombre 91 provinces ou États au total, à savoir 10 au Canada, sans compter trois territoires, le Yukon, les Territoires du Nord-Ouest et le Nunavut, 50 aux États-Unis, sans compter le district de Columbia, c'est-à-dire la capitale fédérale, non plus que Puerto Rico ou les îles Vierges, et 31 au Mexique, sans compter le district fédéral.

Au niveau local, le nombre des gouvernements atteint officiellement 84955 aux États-Unis, où la notion de gouvernement local inclut aussi bien les comtés, les municipalités, les communes et les districts scolaires 
que de nombreux districts spéciaux ${ }^{7}$. Trois critères servent à établir l'existence d'un gouvernement local aux fins du recensement, à savoir: une existence organisée, le caractère gouvernemental des activités exercées et une autonomie substantielle. Tandis que le plus grand nombre de ces gouvernements peut prélever des taxes, plusieurs districts spéciaux, qu'il s'agisse de sociétés publiques d'habitation, d'énergie et le reste, sont financés grâce à l'imposition de tarifs ou de loyers, ou encore par le biais de subventions. En 1992, le nombre des gouvernements locaux se décompose comme suit: 3043 comtés, 19279 municipalités, 16656 communes, 14422 districts scolaires et 31555 districts spéciaux. Si le niveau d'emploi peut constituer un critère significatif, à certains égards, de l'importance relative des trois paliers de gouvernement aux États-Unis, il est intéressant de noter que les gouvernements étatiques et locaux accaparent à eux seuls $84 \%$ du nombre total des employés civils gouvernementaux; ainsi, il y avait, en 1993, la dernière année pour laquelle ce genre de données est disponible, 3 millions d'employés fédéraux, 4,7 millions dans les États et 11,2 millions au niveau local.

Au Canada, la notion d' «administration locale» comprend les entités gouvernementales créées par les provinces et les territoires; elles sont regroupées en fonction de leur rôle premier, à savoir la protection, le transport, l'hygiène, la santé et le bien-être, l'éducation ou l'environnement. L'unité d'administration locale, à l'exception du conseil scolaire, est généralement la municipalité. Au demeurant, on assiste, dans ce cas-ci également, à la création d'un nombre croissant d'organismes spéciaux ou de conseils mixtes ${ }^{8}$. On dénombrait, au total, 4634 municipalités à la fin des années quatre-vingt, dont $4238 \mathrm{mu}$ nicipalités proprement dites comprenant cités, villes, villages et municipalités rurales, le reste étant composé de municipalités régionales, une expression qui inclut les communautés urbaines comme celles de Montréal, Québec ou Toronto et des quasi-municipalités.

Au Mexique, même si c'est la notion de municipio qui apparaît dans la Constitution, c'est plutôt celle de localidad qui sert de base dans la collecte de certaines données de recensement. Il faut cependant préciser que si leur nombre s'élève à plus de 125000 , en fait, seules les plus importantes parmi ces localités, soit 2376 selon une étude comparative du Fonds monétaire international (FMI) datant de 1988, jouissent d'une existence administrative quelconque.

7 U.S. Bureau of the Census, Statistical Abstract of the United States: 1996, Washington, D.C., 1996, p. 293.

8 Statistique Canada, Annuaire du Canada, Ottawa, Statistique Canada, 1990. 
Mais puisque la notion de gouvernement local regroupe des unités de tailles très diverses, il est sans doute plus pertinent de retenir, pour terminer cette description, que ces trois pays comptent au total à peu près 40000 cités et villes, dont environ 46 zones métropolitaines ayant une population totale supérieure à un million et environ 280 villes de 100000 habitants ou plus 9 .

Il est à la fois intéressant et significatif de souligner, au passage, que cet accroissement spectaculaire du nombre de zones urbaines et de villes importantes est bien sûr le résultat premier de la croissance des populations et de l'extension de l'urbanisation, mais un tel accroissement reflète également un phénomène fort révélateur qui est celui de l'augmentation en nombre et en puissance des pouvoirs locaux euxmêmes. Ce processus est peut-être moins souvent relevé que l'est celui de la croissance du nombre des États souverains depuis la Seconde Guerre $^{10}$, mais il n'en paraît pas moins digne de mention dans la mesure où certaines entités publiques dites «locales», qu'il s'agisse de villes, de métropoles ou de zones urbaines, jouissent d'un poids socioéconomique et bénéficient d'un pouvoir politique qui dépasse, dans bien des cas, celui des États ou provinces dont elles font partie et dont elles relèvent sur le plan constitutionnel.

\subsection{Les gouvernements provincial et municipal selon le rapport Macdonald}

Dans le contexte de l'après-guerre, et à la faveur de l'emprise tout compte fait universelle qu'exercent les paramètres du keynésianisme sur la gouverne des économies et des sociétés, c'est essentiellement le pouvoir central qui se pose comme le régulateur obligé de l'économie et de la société. En ce sens, et comme l'ont noté plusieurs analystes de la scène politique canadienne à l'époque ${ }^{11}$, le keynésianisme se fondait

9 E. H. Fry, art. cité, p. 21.

10 Il y avait 55 pays qui étaient représentés à San Francisco en 1945, et les Nations unies comptent 185 membres aujourd'hui. Cependant, cet indicateur n'est pas le seul ni le plus probant sans doute, puisque plusieurs pays ne sont pas membres de l'ONU, comme la Suisse ou le Vatican, de sorte que le nombre total des pays excéderait les 210 à l'heure actuelle, selon certains analystes. Il serait alors fort intéressant de pouvoir calculer également l'évolution du nombre des gouvernements intermédiaires qui a dî croître de manière encore plus importante pour deux raisons: la première, c'est l'augmentation des gouvernements intermédiaires au sein des vieilles fédérations et, la seconde, c'est l'augmentation du nombre des fédérations elles-mêmes.

11 Par exemple, F.-A. Angers, Essai sur la centralisation. Analyse des principes et perspectives canadiennes, Montréal, Beauchemin, 1959, au chap. VI: «Keynes et le problème de la centralisation des pouvoirs», chapitre rédigé par P. Harvey et J. Parizeau. 
sur la centralisation des instruments de la gestion de l'économie politique au pays et a contribué, de ce fait, à une révision en profondeur des relations entre les niveaux fédéral et provinciaux de gouvernement. Cette révision aura lieu très tôt, c'est-à-dire pendant la Seconde Guerre, alors que les gouvernements des provinces consentiront à la modification constitutionnelle sollicitée par le fédéral, en vertu de laquelle le chômage devient un domaine de compétence fédérale et, indirectement, la main-d'œuvre, qui est, comme on le sait, un élément essentiel dans la poursuite de cette économie politique inspirée de la Théorie générale de l'emploi, de l'intérêt et de la monnaie de John Maynard Keynes. Si les provinces consentent à ce transfert à ce moment-là, c'est essentiellement à cause de la situation d'urgence créée par la guerre elle-même, bien sûr, mais ce serait également parce que les dirigeants fédéraux auraient laissé entendre que la question serait de nouveau débattue à la fin des hostilités. C'est ce qui explique que les débats reprendront de plus belle à la fin des années quarante et tout au long des années cinquante. Or, si l'instauration d'une politique économique keynésienne attise et alimente les affrontements et les escarmouches entre le pouvoir fédéral et les provinces, elle n'en contribue pas moins à marginaliser à peu près complètement le troisième et dernier niveau de pouvoir, le pouvoir municipal.

Cependant, comme le rappelait avec justesse K. G. Crawford dans sa préface à la première édition de son ouvrage classique sur le gouvernement municipal au Canada, «the student who would understand the government of a country can only obtain a complete knowledge of it if he knows something of the government at its local or municipal level ${ }^{12}$ ». Or, quand on pense à l'ensemble des politiques d'emploi, des politiques sociales et des différents régimes de santé ou d'éducation ou de services sociaux qui forment et constituent cet Étatprovidence et qui, de ce fait, entretiennent ces rivalités entre niveaux de gouvernement plus ou moins souverains dans tel ou tel champ de compétence, on ne prend pas en compte le fait que les nombreuses missions propres à cet État-providence sont réalisées, parallèlement et, parfois, concurremment, par des administrations qui relèvent de niveaux de gouvernement différents. Et quand cette réalité est prise en considération, dans le contexte canadien, en particulier, trop souvent on se contente d'envisager l'État-providence comme un complexe de

Sur cette question, et sur le débat qu'elle a soulevé entre les tenants et les adversaires du keynésianisme, débat qui s'est cristallisé un temps autour de l'ouvrage de M. Lamontagne (Fédéralisme canadien, Québec, Presses de l'Université Laval, 1954) que défendait, en particulier, P. E. Trudeau, voir D. Brunelle, Les trois colombes, Montréal, VLB éditeur, 1985.

12 K. G. Crawford, Canadian Municipal Government, Toronto, University of Toronto Press, 1954, p. IX. 
rapports et de relations entre deux niveaux de gouvernement, les pouvoirs fédéral et provincial, sans lever le voile sur les conséquences que ce régime d'État a pu produire au niveau municipal ou local. Nous voyons pourtant à quel point cette réduction des rôles et fonctions de l'Etat à deux instances ou à deux niveaux, au lieu des trois qui existent en droit et en fait, constitue une omission pour le moins délicate, quand on rappelle simplement ce fait historique que ce fut précisément pour faire face à l'endettement des pouvoirs locaux, submergés par l'accroissement de leurs dépenses sociales au moment de la crise économique des années trente qu'ont été prises en charge par les niveaux supérieurs de gouvernement des responsabilités auparavant assumées par ces mêmes gouvernements locaux ${ }^{13}$. En ce sens, l'Étatprovidence, dans le contexte canadien surtout, en légitimant et en opérant une vaste appropriation de pouvoirs auprès des gouvernements locaux et en effectuant une nouvelle redistribution asymétrique de ces pouvoirs entre les gouvernements provinciaux et fédéral, s'il préparait le terrain sur lequel allaient s'affronter ces deux partenaires à l'avenir, contribuait, par la même occasion comme nous venons de le souligner, à une marginalisation irrémédiable des gouvernements locaux euxmêmes.

Cela veut dire, en d'autres termes, que l'on ne pourra pas comprendre la gouverne politique dans son ensemble, son cadre de référence, sa logique ou, mieux, ses logiques d'action, comme ses finalités, sans accorder toute son importance au fait que, en régime fédéral, nous avons bel et bien affaire à trois niveaux de gouvernement et non pas à deux comme on l'infère souvent. Cette délimitation entre trois niveaux est décisive, et ce malgré le fait que la Constitution de 1867 n'accorde pas au gouvernement municipal de souveraineté en propre, comme elle le fait pour les deux autres paliers, puisque les villes et municipalités sont sous l'autorité des gouvernements provinciaux. À son tour, cette répartition n'est pas sans conséquences, puisqu'elle implique maintenant qu'il faille composer avec «dix systèmes différents de gouvernements locaux ${ }^{14} \gg$ dans le contexte constitutionnel canadien.

Or, la mise en œuvre et l'opérationnalisation d'une économiepolitique inspirée de Keynes servira de base à une nouvelle délimitation des pouvoirs et responsabilités entre le fédéral et les provinces grâce à l'amendement constitutionnel qui transférera au gouvernement fédéral

13 Comme le souligne K. G. Crawford, durant les années trente, les municipalités ont été contraintes de procéder à des réductions «drastiques» dans la prestation de services et plus de 50 d'entre elles se sont retrouvées en défaut de paiement vis-à-vis de leurs obligataires afin de faire face aux dépenses de bien-être social et de l'assistance aux sans-emploi (ibid., p. 341).

14 Ibid., p. ix. 
la compétence en matière d'assurance-chômage ${ }^{15}$. Ce simple amendement confiait en effet au gouvernement fédéral les instruments indispensables à la poursuite de la croissance économique et confinait les provinces dans leurs missions sociales. À son tour, cette révision du cadre d'analyse et d'intervention publique devait conduire, au-delà de la redéfinition du contenu d'un partage de responsabilités entre les ordres principaux de gouvernement, à la révision des rôles et fonctions assumés par les gouvernements locaux ${ }^{16}$.

Étant donné l'importance du rapport de la commission Macdonald dans la redéfinition des paramètres de la gouverne politique au pays et le rôle central de ses recommandations dans le largage des paramètres du keynésianisme d'un côté, dans le recours au libre-échange de l'autre, il nous semble intéressant de rappeler brièvement la teneur des arguments concernant la question des pouvoirs provincial et local.

La prévision du rapport est claire à ce propos: elle établit qu'un éventuel libre-échange canado-américain «obligerait les provinces à abandonner une partie de leur liberté en ce qui concerne l'utilisation des politiques portant sur les barrières non tarifaires, [une] contrainte [qui] les priverait sans doute d'une partie de leur champ de manœuvre politique et gouvernementale ${ }^{17}$ ». Néanmoins, au-delà de ce constat, il n'en demeure pas moins que l'instauration d'une intégration économique à grande échelle exige une révision profonde des formes et modalités de l'intégration économique domestique. C'est au volume 3 du rapport que l'on trouve les analyses les plus pertinentes sur le défi que constitue le maintien de l'«union économique» dans un contexte caractérisé par l'accroissement de l'intégration dans un axe nord-sud. De manière à peine provocante, les commissaires entendent faire face à ce qu'ils désignent comme une menace de «balkanisation ${ }^{18}$ de

15 Il s'agit de l'amendement du 10 juillet 1940 qui ajoute un paragraphe 2a à l'article 91 de l'Acte de l'Amérique du Nord britannique de 1867.

16 Un indice révélateur de la difficulté d'encadrer les relations entre les pouvoirs provinciaux et leurs municipalités nous est fourni par le nombre élevé de commissions d'études et d'enquêtes nommées par les provinces pour étudier ces questions entre les années trente et les années cinquante: Alberta en 1935 et 1948; Saskatchewan en 1936 et 1950; Colombie-Britannique en 1947; Nouvelle-Écosse en 1949; Ontario et Manitoba en 1951 (K. G. Crawford, ouvr. cité, p. 335, note 1).

17 Rapport de la Commission royale sur l'union économique et les perspectives de développement du Canada, ouvr. cité, vol. 1, p. 401.

18 Ibid., vol. 3, p. 110, 148, 150. Cette métaphore se trouvait déjà dans un rapport diffusé par le Conseil économique de l'Ontario (voir M. J. Trebilcock et autres, Federalism and the Canadian Economic Union, Toronto, Ontario Economic Council, 1983, p. 48). Il n'est sans doute pas inutile d'ajouter que l'expression a été utilisée au départ par des auteurs marxisants avant de passer dans des discours appartenant à la nébuleuse libérale. 
l'espace économique canadien et, à cette fin, ils se penchent, entre autres choses, sur la question des «restrictions internes» aux échanges. À leurs yeux, «les gouvernements provinciaux créent des distorsions en imposant des limites provinciales au commerce ${ }^{19}$ ». Ils citent à ce propos l'Association canadienne des manufacturiers (ACM) qui estime que la prolifération des limitations imposées par les provinces ralentit la croissance du commerce interprovincial. Afin de relever ce défi, les commissaires envisagent de «renforcer la protection constitutionnelle de l'union économique, [qui] devrait se faire au moyen d'un amendement à la Constitution ${ }^{20} \gg$. Il s'agirait alors de modifier l'article 121 qui prévoit déjà la libre circulation intérieure des biens, de manière à offrir une protection constitutionnelle contre les barrières tarifaires, et d'étendre son application à la circulation des services. Cependant, avant d'en arriver là, et parce que cette recommandation risque d'aller à l'encontre «des autres intérêts de la fédération», les commissaires recommandent plutôt aux gouvernements fédéral et provinciaux de s'efforcer de mettre au point un «code de conduite économique» qui représente «un processus d'implantation circonspect et progressif» sans doute plus adapté aux circonstances à la fois fluctuantes et volatiles qui caractérisent les relations entre les provinces et le fédéral, comme quoi on envisage un processus à la fois long et délicat en vue d'en arriver à l'union économique souhaitée. En ce sens, compte tenu des contraintes propres à la diplomatie intérieure au Canada, on préfère la négociation permanente à la modification de la Constitution qui aurait pour effet premier de confier aux tribunaux le règlement de questions relevant de la politique économique intérieure.

Poussant plus avant la réflexion, le rapport, après avoir rappelé que le fédéralisme canadien est fondé sur «la répartition des compétences entre deux ordres de gouvernement, [que], dans sa propre sphère, chaque gouvernement est souverain ${ }^{2 l_{1}}$ et que les administrations locales sont des créations des gouvernements provinciaux, fait remarquer que certaines administrations locales sont plus importantes que des gouvernements provinciaux eux-mêmes, comme c'est le cas pour les agglomérations de Montréal et de Toronto qui, toutes deux, représentent plus de la moitié de la population de leurs provinces respectives. Afin de tenir compte de cette nouvelle réalité, de même que des entraves inscrites dans le mode antérieur de partage des responsabilités entre les niveaux, les gouvernements provinciaux ont institué, au-delà des administations locales, un nouvel ordre de gouvernement intermédiaire qui constituerait en quelque sorte un quatrième niveau ou ordre de

19 Rapport de la Commission royale sur l'union économique et les perspectives de développement du Canada, vol. 3, p. 130.

20 Ibid., p. 151.

21 Ibid., vol. 1, p. 15. 
gouvernement, comme c'est le cas pour les communautés urbaines et, pourrions-nous ajouter, comme c'est le cas aussi pour les municipalités régionales de comté (MRC) au Québec.

Pourtant, même si le rapport Macdonald avait souligné «l'incertitude du statut des administrations locales et la complexité de leur cadre législatif et institutionnel, [leur] enlis[ment] dans des détails administratifs», de même que la plus grande accessibilité de leur processus décisionnel au public, par opposition à la difficulté d'accès dans les deux autres ordres, les commissaires devaient rejeter l'option d'une reconnaissance en bonne et due forme du statut constitutionnel des administrations locales ${ }^{22}$. Cette reconnaissance avait été revendiquée par certaines villes elles-mêmes, Windsor par exemple, essentiellement comme une mesure susceptible, d'une part, d'accroître la marge de manœuvre politique des villes face à des demandes de plus en plus contradictoires issues du gouvernement provincial et des milieux d'affaires, et, d'autre part, de donner une plus grande marge de sécurité financière dans un contexte de déséquilibre croissant entre revenus et dépenses $^{23}$. Face à cette revendication, le rapport se contente de renvoyer la responsabilité au gouvernement provincial afin qu'il examine «la possibilité d'adopter une procédure spéciale pour modifier les fonctions attribuées aux collectivités locales ${ }^{24}$ ».

L'augmentation des dépenses des administrations municipales ne laisse pas d'inquiéter les commissaires et ils soulignent à ce propos que, déjà au début des années quatre-vingt, «les municipalités dépensent plus de deux fois le montant qu'elles perçoivent en taxes», d'où leur situation de dépendance croissante à l'endroit «de sources de revenus qui échappent à leur contrôle et, par conséquent, [une] incertitude au sujet du montant des fonds qu'elles recevront au cours de l'année ${ }^{25}$ ». Le rapport développe ensuite cette argumentation et met alors en lumière un problème central:

Le système de transfert tripartite que nous avons au Canada rend l'imputabilité très difficile, surtout lorsqu'une grande partie des dépenses engagées par un ordre de gouvernement sont payées par des taxes imposées par un autre ordre de gouvernement. Cette question d'imputabilité se complique encore au niveau local en raison de la façon dont les subventions provinciales sont versées. Depuis quelques années, dans toutes les provinces, les subventions aux municipalités ont été de plus en plus souvent assujetties à des conditions [...].

Les administrations locales nous ont fait part des conséquences néfastes du

22 Ibid., vol. 3, p. 549.

23 Ibid., p. 433.

24 Ibid.

25 Ibid., p. 437. 
système de subventions conditionnelles, tant sur leur imputabilité que sur le contrôle des finances publiques au pays ${ }^{26}$.

Parmi les solutions ou recommandations envisagées par la commission, trois méritent d'être signalées: une première concerne l'attribution d'une partie déterminée des recettes de l'impôt sur le revenu ou de la taxe de vente au financement des administrations locales, une deuxième porte sur l'attribution de l'élaboration et de la mise en œuvre des politiques d'urbanisation aux administrations locales et la troisième propose la simplification des ententes juridiques et institutionnelles, ainsi que l'établissement de communications claires et directes entre pouvoirs décisionnels locaux et provinciaux ${ }^{27}$.

Il convient de noter, pour terminer cette présentation sur une note plus critique, que la commission Macdonald a fait preuve d'une retenue excessive en la matière, voire d'irréalisme, en renvoyant la question au palier provincial, alors qu'il aurait fallu dénoncer la stratégie privilégiée par les autres niveaux de pouvoirs, qui consiste à mettre sur pied des agences et autres entités publiques provinciales ou fédérales à l'échelle locale, au lieu d'accroître et d'étendre les prérogatives (empowerment) des acteurs locaux et de leurs représentants. Déjà, au cours des années trente, dans un ouvrage magistral ignoré aujourd'hui malgré ses nombreuses rééditions jusque dans les années soixante, le juriste anglais C. K. Allen avait dénoncé l'irréalisme dont font preuve maints constitutionnalistes quand ils ignorent ou escamotent l'important phénomène que l'on désigne, en droit britannique, sous le nom de devolution, c'est-à-dire la décentralisation ou la déconcentration administrative ${ }^{28}$. Cette approche conduit les pouvoirs exécutifs «souverains» à recourir de plus en plus systématiquement à une législation déléguée en vertu de laquelle ce sont des entités administratives placées sous leur contrôle propre qui assurent la production et la distribution de services publics et sociaux à l'échelle locale. C'est ainsi que l'on a assisté à la prolifération des conseils, sociétés et autres bureaux administratifs qui gèrent tout un ensemble de politiques et de programmes dans des domaines comme la santé, l'éducation et les services sociaux. Or cette décentralisation n'est pas sans avoir des répercussions sur les pouvoirs des municipalités ellesmêmes, ainsi que sur l'ensemble de la gestion locale des services sociaux. Dans ces conditions, l'enjeu de l'imputabilité ne devrait pas prendre à partie le seul niveau municipal de gouvernement et d'administration, comme si ce pouvoir opérait encore et toujours dans un environnement parfaitement délimité et délimitable, il devrait aussi

26 Ibid., p. 437-438.

27 Ibid., p. 440-441

28 C. K. Allen, Law in the Making, Oxford, Oxford University Press, 1960. 
s'étendre à ces autres entités administratives locales qui répondent bien plus souvent de leurs mission et mandat auprès des ordres supérieurs de gouvernement qu'ils n'en répondent au niveau local même, avec tous les effets pervers qu'une telle asymétrie des imputabilités ne manque pas de causer. Cela dit, nous aurons l'occasion de revenir sur l'enjeu de la réforme au niveau régional.

\subsection{Libre-échange et constitutionnalisme}

Le recours au libre-échange s'inscrit dans une stratégie nouvelle qui conduit les États à favoriser, par ce biais, la compétitivité de leurs entreprises transnationales, et partant, l'intégration compétitive des économies nationales à l'économie mondiale ${ }^{29}$. À cette fin, les gouvernements centraux sont amenés à revoir les termes et les modalités de leurs interventions passées dans l'économie et la société. À son tour, cette révision a deux effets majeurs: elle oblige le gouvernement à redéfinir les paramètres de ses propres interventions et à revoir son rapport avec les autres niveaux de gouvernement. En d'autres termes, le libre-échange, en tant que politique économique susceptible de modifier les formes et contenus des interventions antérieures, conduit les gouvernements à entreprendre une révision constitutionnelle ou quasi constitutionnelle des partages de responsabilités et de prérogatives entre les ordres de gouvernement. Nous aurions donc un indicateur significatif de l'importance que revêt le libre-échange dans la redéfinition d'une économie politique nationale si le recours à cette mesure était soit précédé ou suivi de modifications à la Constitution même du pays qui l'adopterait, voire d'une quelconque redéfinition des pouvoirs entre niveaux de gouvernement, que ce soit entre les pouvoirs centraux et les États ou provinces, entre ces derniers et les gouvernements locaux.

On peut ainsi se faire une idée de l'ampleur des réaménagements consécutifs à la sanction du régionalisme économique dans les Amériques une fois souligné le fait que plusieurs des États fédérés qui sont parties à de tels accords procèdent ou ont procédé à des réformes constitutionnelles majeures dont certains aspects touchent précisément à la redéfinition des relations entre le pouvoir central et les unités infraétatiques. En effet, la signature de l'ALE, de l'ALENA et du MERCOSUR a été, dans les trois cas, précédée ou suivie de révisions significatives des textes fondateurs, tant au Canada, au Mexique, au Brésil qu'en Argentine. Au Canada, cela s'était produit en 1982, trois ans avant l'ouverture des négociations bilatérales dans la foulée du

29 C. Deblock, «Du plein-emploi à la compétitivité», dans F. Crépeau (dir.), Mondialisation des échanges et fonctions de l'État, Bruxelles, Brylant, 1997, p. 63122. 
rapatriement de la Constitution et de la promulgation du Canada Bill. Dans ce cas-ci, comme on vient de le voir, le processus s'étire au-delà de cet événement, puisque nous sommes toujours aux prises avec les séquelles des recommandations du rapport Macdonald. Parfois, comme ce fut le cas au Mexique, le lien entre une modification à la Constitution et l'ALENA n'avait pas été rendu explicite, de sorte que ce n'est qu'après le fait que les analystes ont pu établir la relation entre la modification apportée à l'article 27 de la Constitution du Mexique en 1991, qui accordait au tenancier la propriété pleine et entière de l'ejido, et les exigences liées à l'accroissement de la productivité agricole consécutives à la signature de l'accord ${ }^{30}$, un lien qu'avaient bien sûr établi les zapatistes, ce qui explique pourquoi ils avaient résolu d'intervenir sur la scène médiatique mondiale précisément le jour de l'entrée en vigueur de l'ALENA, le 1er janvier 1994.

Cependant, la question des effets des accords sur les entités infraétatiques ne saurait être envisagée à partir du seul angle d'approche formel et juridique selon lequel il suffirait de repérer les modifications faites aux constitutions visant la répartition des pouvoirs entre les niveaux de gouvernement. Car les effets éventuels de la négociation de certains accords internationaux sur les unités constituantes dépendent également de la nature du processus de mise en œuvre des termes des accords dans chacune des fédérations. Dans les cas américain et canadien, les accords internationaux ne font partie de la législation nationale qu'à compter du moment où le Sénat ou le Parlement en ont repris les termes et dispositions dans une loi nationale. Au Mexique, en revanche, le contenu des accords internationaux est intégré à la législation nationale dès la signature. Nous avons affaire dans les premiers cas à deux pays qui opèrent encore et toujours sous l'égide du monisme juridique, tandis que le troisième participe d'un pluralisme juridique tel qu'on le rencontre, par exemple, en Europe communautaire où les normes européennes sont intégrées au droit interne, encore que le Mexique se distingue des autres pays qui sanctionnent le pluralisme juridique en ce que les normes internationales auxquelles il souscrit, à l'instar d'une panoplie d'autres normes d'ailleurs, ne sont pas nécessairement appliquées.

Aux États-Unis, le Sénat dispose seul des prérogatives en matière d'adaptation et d'intégration des termes des traités et autres accords internationaux au droit interne. Au Canada, le gouvernement fédéral ne dispose pas du pouvoir d'engager les provinces, le résultat, confirmé par

30 R. Delgado Moya et L. Hernandez Ramirez, «Sector agropecuario, medidas sanitarias y fitosanitaarias del tlcan en la legislacion nacional», dans J. Witker (dir.), El Tratado de Libre Comercio de América del Norte. Analisis, diagnostico y propuestas juridicos, México, Universidad Nacional Autonoma de Mexico, 1993, p. 283. 
les tribunaux d'ailleurs, étant que, si le gouvernement fédéral peut signer certains accords ou traités dont la matière relève de pouvoirs dévolus aux provinces en vertu de la Constitution, les provinces ne sont pas tenues par les termes de ces accords tant et aussi longtemps qu'elles ne les ont pas sanctionnés dans une loi provinciale, ou par décret, comme l'avait fait le gouvernement québécois sous le premier ministre Bourassa à propos de l'Accord de libre-échange entre les États-Unis et le Canada. En ce sens, la situation au Mexique et en Amérique latine est passablement différente de celle qui caractérise les États-Unis et le Canada, puisque, même là où, en principe, la Constitution a instauré un système fédéral, en pratique, c'est le pouvoir central qui a la haute main sur la gouverne du pays, quelle que soit la répartition des pouvoirs inscrite dans l'acte fondateur. C'est ainsi que les quatre fédérations de l'Amérique latine, le Mexique, le Venezuela, le Brésil et l'Argentine, vivent pour ainsi dire sous l'empire de régimes unitaires et centralisateurs, et ce malgré que les textes constitutionnels aient été fortement inspirés par le modèle américain. Ce qui ne veut évidemment pas dire qu'on ne s'y préoccupe pas de développement provincial ou estatal selon les cas, mais ce qui veut dire que les initiatives sont fortement marquées par la centralisation des politiques économiques nationales. Mais cela est sans doute en passe d'être remis en cause, si l'on en juge par les récentes modifications apportées à la Constitution de l'Argentine qui, depuis 1994, reconnaît aux gouvernements provinciaux le droit d'instituer des zones franches pour attirer les investisseurs étrangers, une ouverture dont s'est prévalue depuis la province de Santa $\mathrm{Cruz}^{31}$.

Enfin, au-delà des effets de débordement dans le domaine constitutionnel, d'une part, et de l'autonomie relative des entités infraétatiques face à l'exercice de leur souveraineté internationale de la part des États centraux de l'autre, il y a deux autres dimensions des conséquences du libre-échange sur les États et provinces qu'il convient de relever à ce stade-ci. L'une porte sur les formes et modalités de la négociation elle-même, puisque les États centraux font de plus en plus appel, par voie de consultation ou autrement, à une participation plus active de la part des États et provinces, tandis que l'autre dimension renvoie, quant à elle, à la nature des relations entre les niveaux central, intermédiaire ou local de l'État et à leurs liens avec les acteurs sociaux. Ce que l'on cherche à cerner dans ce dernier cas, c'est l'articulation, s'il en est, entre la reconfiguration interne des États et l'instauration des nouveaux partenariats politiques, économiques et sociaux, questions sur lesquelles nous reviendrons. Avant d'aller plus loin, il convient de cerner de manière plus formelle le champ de l'analyse en nous attardant

31 D. J. Martinez Llaneza, «Zona franca y desarrollo de Santa Cruz», dans L. E. Di Marco (dir.), La Argentina Contemporanea, Córdoba, Ediciones CIEC, 1995, p. 248. 
sur les dispositions des deux accords de libre-échange qui ont lié successivement le Canada et les États-Unis, et plus tard, ces deux derniers et le Mexique. Comme nous le verrons, le retour sur l'ALE est indispensable pour comprendre pleinement l'effet de certaines dispositions de l'ALENA sur les pouvoirs des gouvernements infraétatiques.

\section{Les dispositions qui affectent les gouvernements infra-étatiques en Amérique du Nord: de l'ALE à l'ALENA}

Nous allons passer en revue, dans l'ordre, les articles des accords de libre-échange qui touchent directement les prérogatives des provinces ou des États, ainsi que les gouvernements locaux.

En ce qui concerne le premier accord bilatéral (ALE) entré en vigueur en 1989, la disposition centrale à ce propos est sans conteste l'article 103 du chapitre 1, «Objectifs et portée», qui stipule que:

Les parties au présent accord veilleront à ce que toutes les mesures nécessaires soient prises pour donner effet aux dispositions de l'Accord, y compris à leur observance, sauf stipulation contraire dans les présentes, par les gouvernements des États et des provinces et les administrations locales.

On a pu dire de cette formulation qu'elle représentait la clause fédérale la plus forte à laquelle le Canada et, devons-nous ajouter, les États-Unis aient jamais donné leur aval ${ }^{32}$; cela est d'autant plus vrai si l'on prend en considération ce fait incontournable, à savoir que la plupart des thèmes et sujets couverts par l'ALE affectaient, que ce soit de manière principale ou de manière accessoire, les prérogatives des provinces et, dans une moindre mesure, celles des pouvoirs locaux. Pour saisir toute la portée de cette conclusion dans son application au contexte canadien surtout, il faut tenir compte non seulement des articles qui font explicitement référence aux prérogatives des provinces, mais également de ceux qui ne font que les réaffirmer sans plus, une stratégie dont on comprendra toute la valeur quand viendra le temps de négocier un accord subséquent engageant les mêmes partenaires, auxquels s'ajoute le Mexique cette fois, quelques années plus tard, alors que ce seront précisément ces cas d'exception qui feront l'objet de ces nouvelles négociations.

32 D. M. Brown et E. H. Fry (dir.), States and Provinces in the International Economy, Berkeley, Institute of Governmental Studies Press, University of California, 1993, p. 115 . 
Les autres dispositions de l'ALE qui touchent positivement ou négativement aux pouvoirs des provinces et des États se trouvent, en dehors des définitions générales de «province» et d' «État» figurant au chapitre 2 (art. 201, paragr. 1), au chapitre 5 sur le traitement national (art. 502), au chapitre 6 sur les normes techniques, où les provinces et États sont nommément exclus de l'application des dispositions du chapitre à l'article 601, au chapitre 7 sur l'agriculture (art. 701 à 711 , mais surtout dans les appendices 1, paragr. B12, et 2, paragr. A4 et B16), au chapitre 8 sur les vins et spiritueux (art. 802 et 804), au chapitre 9 sur l'énergie (art. 905), au chapitre 12 à propos des exceptions (art. 1203 et 1204), au chapitre 14 sur les services (art. 1402) et, enfin, au chapitre 16 sur l'investissement (art. 1604, paragr. 4, et art. 1611, notamment aux définitions des mots «investisseur» et «société d'État»).

Cependant, ces dispositions n'ont ni la même importance ni la même portée. Par exemple, l'article 502 ne fait qu'étendre aux provinces et États indifféremment la règle du General Agreement on Tarif and Trade (GATT) en matière de traitement national:

Les dispositions du présent chapitre concernant le traitement national devant être accordé aux produits similaires, directement concurrents ou substituables, seront interprétées comme signifiant, dans le cas d'une province du Canada ou d'un État des États-Unis d'Amérique, un traitement non moins favorable que le traitement le plus favorable accordé par cette province ou cet État aux produits similaires, directement concurrents ou substituables, selon le cas, de la Partie sur le territoire duquel elle ou il se trouve.

Comme l'indique le texte d'accompagnement, «ce chapitre assujettit clairement les mesures adoptées par les provinces ou États à l'obligation de traitement national en vertu du GATT. Cela signifie qu'une province ou un État ne peut pas faire de discrimination à l'encontre de produits importés en prenant des mesures qui relèvent pourtant de sa compétence ${ }^{33} \gg$. Par ailleurs, si les dispositions en matière d'agriculture ne viennent pas encore bouleverser un domaine de compétence concurrent relevant des deux niveaux de gouvernement aussi bien aux États-Unis qu'au Canada, il n'en va plus de même en matière de vins et spiritueux (au chap. 8), puisque la vente et la distribution relèvent essentiellement de régies autonomes exploitées par les gouvernements provinciaux au Canada, ce qui n'est le cas que pour 17 Etats aux ÉtatsUnis, ou pour l'énergie (au chap. 9), un autre domaine de compétence provinciale. En revanche, le chapitre 12 comprend un certain nombre

33 Ministère des Affaires extérieures, Accord de libre-échange entre le Canada et les États-Unis. Le commerce: la clé de l'avenir, Ottawa, 1998, p. 68. 
d'exceptions dont celle qui concerne la vente et la distribution intérieures de la bière, domaine qui, lui aussi, est du ressort des provinces. Enfin, il est important de souligner que les deux thèmes majeurs de cette entente, les services et l'investissement (aux chap. 14 et 16), avaient été négociés de manière à engager également les provinces et les États sur la voie de la déréglementation de ces deux marchés.

À la vérité, on peut conclure ce rapide tour d'horizon en soutenant, à l'encontre de l'interprétation défendue par Douglas $M$. Brown pour qui l'accord touchait bien peu, en dehors des dispositions du chapitre 8 , aux prérogatives des provinces ${ }^{34}$, que, dans son esprit comme dans sa lettre, l'ALE représentait ni plus ni moins qu'un accord par lequel les deux gouvernements fédéraux négociaient désormais non seulement à l'intérieur de domaines de compétence partagés, mais bel et bien dans des domaines exclusivement réservés aux provinces ou aux États selon les cas. C'est d'ailleurs ce constat qui permet de donner tout son sens au fait que l'article concernant l'étendue des obligations vienne en troisième place (art. 103), après l'article relatif à l'établissement de la zone de libre-échange (art. 101) et l'article consacré à la définition des cinq objectifs de l'accord (art. 102), une séquence qui montre bien que, d'une part, l'extension aux provinces et aux États faisait partie des priorités des signataires et ne relevait nullement de l'ordre des souhaits ou des intentions et que, d'autre part, ce sont des pouvoirs généraux qui sont visés et non pas tel ou tel programme. C'est en ce sens que nous avons évoqué l'esprit de l'accord au-delà de la seule prise en compte de sa lettre.

On peut prendre une autre mesure de ce constat en relevant que, des deux, ce sont les pouvoirs des provinces qui sont davantage battus en brèche comparativement à ceux des États américains, compte tenu que les premières disposent d'une panoplie plus large de prérogatives que leurs vis-à-vis américains, que ce soit en matière d'agriculture, de production de services ou d'investissements, compte tenu également que, à deux reprises, ce sont bien des pouvoirs provinciaux sur la distribution des vins et spiritueux ainsi que sur les sociétés d'État qui sont désignés dans les textes.

Si nous nous tournons maintenant vers le second accord, l'ALENA, que pouvons-nous dire au sujet des dispositions qui affectent les provinces et les États, que ce soit au Canada ou aux États-Unis, de même qu'au Mexique?

34 «There was very little left in terms of direct and immediate impingement on provincial jurisdiction, saving chapter 8.» (D. M. Brown et E. H. Fry [dir.], ouvr. cité, p. 116.) 
En premier lieu, l'article 105 de l'ALENA reprend les termes de l'article 103 de l'ALE cité plus haut, mais sans mentionner les administrations locales; la disposition qui les concerne se retrouve plutôt au chapitre 2 sur les définitions générales, qui précise, au paragraphe 2: «Aux fins du présent accord, et sauf stipulation contraire, toute mention d'une province ou d'un État comprend les administrations locales.» Les commentaires consignés la dernière fois à propos de la place stratégique qu'occupe le principe d'extension aux gouvernements infra-étatiques dans l'accord s'appliquent de nouveau dans ce cas-ci, et il faudrait aussi ajouter, comme on vient de le voir, que l'application du principe s'étend désormais aux administrations locales. L'annexe 201.1 va dans le même sens en précisant les significations de la notion de «territoire» pour chacune des trois parties en présence.

En deuxième lieu, nous retrouvons, aux chapitres correspondants, les mêmes extensions qui ont été soulignées à propos de l'ALE et, en particulier, celle qui apparaît au chapitre 3 sur le traitement national et l'accès aux marchés pour les produits (art. 301), au chapitre 12 sur le commerce transfrontière des services (les art. 1202 «traitement national» et 1213 «définitions») et au chapitre 14 sur les services financiers (art. 1407, paragr. 8, avec réserve à l'art. 1412).

En troisième lieu, enfin, soulignons, parmi les nouvelles dispositions, celle qui se rapporte à la définition d' «une mesure de réglementation de l'énergie [qui] s'entend de toute mesure prise par des entités fédérales ou infranationales et ayant un effet direct sur le transport, la transmission, la distribution, l'achat ou la vente de produits énergétiques ou de produits pétrochimiques de base» (art. 609), l'article 761 du chapitre 7 sur les points d'information concernant les mesures sanitaires ou phytosanitaires adoptées au niveau fédéral, provincial ou de l'État, de même que l'importante substitution à propos des mesures normatives prévues au chapitre 9, en vertu de laquelle l'article 902, paragraphe 1, suspend l'application du principe général d'extension des obligations stipulé à l'article 105 où il s'agissait de «veiller à ce que toutes les mesures nécessaires soient prises pour donner effet aux dispositions du présent accord [...] par les gouvernements des États et des provinces», et lui substitue un engagement beaucoup plus fort de la part des pouvoirs centraux au paragraphe 2 qui édicte:

Chacune des parties s'efforcera, par l'adoption des mesures appropriées, de faire en sorte que les gouvernements provinciaux ou d'État et les organismes non gouvernementaux de normalisation observent les articles 904 à 908 inclusivement sur son territoire.

Pour montrer l'importance de cette disposition, on doit souligner trois choses: d'abord qu'il s'agit désormais de confier au pouvoir 
central la responsabilité d'adopter les mesures appropriées de normalisation, ensuite qu'il s'agit d'instaurer la norme internationale existante comme véritable norme de base (art. 905), enfin, que la normalisation et l'harmonisation s'étendent également aux organismes non gouvernementaux.

Il convient de relever également que le chapitre 10, intitulé «Nouvelles négociations», prévoit l'ouverture des marchés des «entités publiques des provinces et des États» (art. 1002), une ouverture qui est liée à l'implication directe des gouvernements des provinces et des États (annexe 1002.2) à la suite de consultations à venir entre ceux-ci et les gouvernements centraux (art. 1024). Dans le même ordre d'idée, le chapitre 15, «Politique de concurrence, monopoles et entreprises d'État», s'applique tout autant aux entreprises d'État fédérales qu'aux entreprises d'État (annexe 1505.1)

Nous pouvons conclure cette section en soulignant qu'en l'espace d'à peine cinq ans, de 1989 à 1994, les deux accords de libre-échange passés en revue étendent progressivement le degré d'implication des gouvernements infra-étatiques dans le processus d'intégration économique à l'échelle continentale. Pour appuyer davantage cette conclusion, il faudrait nous attarder sur les deux accords parallèles, l'un sur le travail (l'Accord nord-américain de coopération dans le domaine du travail [ANACT]), l'autre sur l'environnement (l'Accord nord-américain de coopération dans le domaine de l'environnement [ANACE]), qui interpellent encore davantage les gouvernements provinciaux et ceux des États fédérés, mais un tel examen, pour aussi significatif et révélateur qu'il soit aux termes de l'argumentation soutenue en ces pages, nous conduirait trop loin et allongerait indûment l'analyse. Quoi qu'il en soit, si les deux accords étudiés ont pour effet d'impliquer les gouvernements infra-étatiques dans le processus d'intégration économique à l'échelle continentale, cela ne doit pas nous amener à sousestimer le fait que ce processus n'a pas seulement pour effet d'engager ces derniers sur le plan «international», il les engage aussi sur un plan interne. Il est en effet de moins en moins possible de distinguer les différents niveaux d'intégration, en particulier les niveaux continental, national et «régional». Le cas du Canada est à cet égard d'autant plus révélateur que, pour des raisons qui tiennent tout autant à son histoire qu'à sa géographie, le marché intérieur ne présente pas le même degré d'unité et de complémentarité économiques que le marché américain, ce qui est assez paradoxal quand on juxtapose ce fait à la vigueur de l'engagement des autorités fédérales dans le libre-échange. Cependant, il ne fait pas de doute que le libre-échange canado-américain a remis à l'ordre du jour les carences de l'intégration au niveau même de l'économie domestique, d'où il résulte que, l'un poussant l'autre en quelque sorte, quelques mois à peine après l'entrée en vigueur de 
l'ALENA, les gouvernements du Canada, des 10 provinces et des Territoires du Nord-Ouest et du Yukon signaient, le 18 juillet 1994, l'Accord sur le commerce intérieur (ACI), accord qui est entré en vigueur le 1er juillet $1995^{35}$. Cet accord est important à double titre: 1) parce qu'il vise à éliminer les obstacles au commerce, aux investissements et à la mobilité intérieure dans les 10 secteurs économiques couverts, celui de l'énergie n'ayant pas encore été inclus, et 2) parce qu'il vise à sanctionner sur le plan juridique une intégration dite en profondeur (deep integration) en engageant formellement les parties contractantes, de même que les paliers de gouvernement inférieurs, les sociétés d'État et les organismes non gouvernementaux, ce qui inclut, entre autres, les municipalités, les établissements d'enseignement supérieur, les écoles ou encore les services de santé financés par l'État ${ }^{36}$. En ce sens, à cause de la manière dont il a été négocié par des parties qui, sans être souveraines, disposent de pouvoirs constitutionnels importants, l'ACI anticipe sur les négociations à venir dans les Amériques dans les domaines qui, d'une manière ou d'une autre, exigeront une concertation de plus en plus étroite entre le gouvernement central et les gouvernements infra-étatiques.

35 L'accord repose sur six principes généraux: la non-discrimination réciproque; le droit d'entrée et de sortie pour les personnes, les marchandises, les services et les investissements; l'absence d'obstacles au commerce intérieur; une définition étroite et non discriminatoire des objectifs légitimes en matière de politiques publiques; la conciliation et l'harmonisation des règlements et normes; la transparence en matière de textes officiels et communications d'avis. Les secteurs économiques couverts sont les suivants: les marchés publics; les investissements; la mobilité de la main-d'œuvre; les mesures et normes en matière de consommation; les produits agricoles et les produits alimentaires; les boissons alcooliques; la transformation des ressources naturelles; les communications; les transports; la protection de l'environnement. La mise en œuvre de l'accord, sa supervision et la conciliation des différends relèvent du Comité du commerce intérieur, composé de représentants de chacune des parties contractantes et ayant rang ministériel. Deux mécanismes de règlement des différends sont également prévus, selon que le différend concerne deux gouvernements ou une personne et un gouvernement.

36 L'une des conséquences de l'ACI est d'éliminer les mesures à caractère préférentiel, dans l'octroi des contrats publics notamment. Ainsi les fournisseurs canadiens peuventils désormais soumissionner sur pratiquement tous les marchés gouvernementaux d'une valeur supérieure à $25000 \$$ pour les produits et à $100000 \$$ pour les services et travaux de construction. Au Québec, la loi 137 adoptée en juin 1997 par l'Assemblée nationale oblige désormais les municipalités à procéder à des appels d'offres publics pour tout contrat de services professionnels de 100000 \$ et plus (voir K. Lévesque, Le Devoir, 23-24 août 1997, p. A3). 


\section{Les défis de l'intégration économique et les nouvelles pratiques}

Après avoir, dans les deux sections précédentes, fait le tour de quelques questions d'ordre général et présenté dans ses grandes lignes le cadre normatif qui fixe les relations à venir entre niveaux de gouvernement, dans la présente section, nous allons modifier l'angle d'analyse et nous attacher désormais à l'étude des ordres ou des niveaux de gouvernement afin de mettre en lumière quelques-unes des principales transformations ou innovations dans leurs pratiques respectives dans la foulée des changements qu'ont entraînés l'intégration économique continentale et, de manière incidente, l'intégration à l'échelle hémisphérique.

Il convient de distinguer à cet égard trois modèles simples de transformations ou d'innovations: celles qui touchent les relations et interrelations verticales entre niveaux ou ordres de pouvoirs, celles qui engagent les relations horizontales entre pouvoirs placés à un même niveau ou pouvoirs appartenant à un même ordre de gouvernement et, enfin, celles qui conduisent les gouvernements à nouer des relations obliques ou transversales entre niveaux ou ordres, ce que Rosenau désigne comme «mixing micro-macro ${ }^{37}$ ». Ce découpage d'apparence simple se complique immédiatement quand il faut prendre en considération le fait que ces relations et interrelations peuvent être engagées tout autant à l'intérieur d'un pays qu'entre pays. Faute de pouvoir rendre compte de toute cette complexité dans ces pages, nous nous contenterons de donner quelques exemples qui nous sont apparus révélateurs des changements en cours. Ces exemples portent sur les dimensions horizontale et verticale uniquement et ne concernent que les provinces et États ${ }^{38}$.

Cependant, avant d'aller plus loin, il convient d'apporter deux brèves précisions. Premièrement, dans le contexte nord-américain plus particulièrement, il n'a pas fallu attendre la sanction d'accords de libreéchange pour assister au resserrement des liens latéraux ou horizontaux directs entre provinces et États voisins, puisque bon nombre de comités, conférences ou réunions transfrontaliers, entre les États-Unis et le Canada surtout, ont été, depuis de nombreuses années, mis sur pied autour d'enjeux comme l'environnement, les migrations ou les

37 J. N. Rosenau, Turbulence in World Politics. A Theory of Change and Continuity, Princeton, Princeton University Press, 1990, p. 141-177.

38 Le lecteur intéressé par les conséquences de ces transformations au niveau local pourra consulter: Y. Bélanger, D. Brunelle et C. Deblock, Relance industrielle et concertation: le cas de la MRC du Bas-Richelieu, 50 p., 1998 (non publié). 
échanges culturels ${ }^{39}$. Deuxièmement, l'établissement des relations horizontales entre gouvernements provinciaux au Canada ou étatiques aux États-Unis remonte à plusieurs décennies, comme c'est le cas pour les Conférences des premiers ministres des provinces du Canada, qui furent instituées en 1926, ou la création de regroupements ou d'associations nationales comme le Council of State Governments aux États-Unis. De plus, aussi bien au Canada qu'aux États-Unis, premiers ministres ou gouverneurs se rencontrent sur une base régionale entre États de l'Ouest, de l'Est ou des Grands Lacs. Enfin, il faut aussi tenir compte d'un phénomène plus récent, celui des associations ou regroupements qui rassemblent États et provinces au niveau transnational, comme le Council of States Governments-Eastern Regional Conference qui regroupe les États du nord-est des États-Unis ainsi qu'un «associé international», la province de Québec, ou encore le Conseil des gouverneurs des Grands Lacs et de l'Ontario qui se réunit annuellement et qui a, en 1997, accueilli le Québec. Une réunion s'est tenue les 10 et 11 juillet 1998 à Érié, en Pennsylvanie, avec les gouverneurs de la Pennsylvanie, du Wisconsin, de l'Indiana, de l'Illinois, du Minnesota, de New York, du Michigan et de l'Ohio. Enfin, on peut relever qu'il existe un conseil semblable sur la côte ouest rassemblant également des représentants des États et des provinces.

Ces deux précisions ne simplifient rien, au contraire, puisqu'elles montrent bien que l'on ne saurait établir de distinction nette entre des types de pratiques dont les uns participeraient des formes actuelles de la continentalisation et les autres, pas. En effet, rien n'empêche les gouvernements d'avoir recours à telle ou telle instance, à un conseil ou à une conférence annuelle, pour redéfinir ou définir des stratégies communes face à l'un ou l'autre aspect de l'intégration économique continentale, et ils $\mathrm{y}$ ont recours régulièrement d'ailleurs, comme l'illustre bien le rôle que la Western Governors Association a joué dans la critique de l'AMI aux États-Unis.

En conséquence, quand nous évoquons des interventions originales au niveau horizontal, nous avons en tête certains phénomènes récents qui semblent ouvrir la voie à de nouvelles modalités d'implication des provinces et États dans le régionalisme économique et dans la continentalisation de leur économie. Nous pouvons citer en exemple la création de l'Association des États du golfe du Mexique, en janvier 1992, une association rassemblant 10 États, cinq au Mexique (Tamaulipas, Veracruz, Tabasco, Campeche et Yucatán) et cinq aux États-Unis (Floride, Alabama, Louisiane, Texas et Mississippi), pour développer les échanges dans la région. Il convient de noter, au passage,

39 Bien sûr, il existe également toute une panoplie de comités transfrontaliers qui sont responsables de la gestion de biens communs, comme les plans d'eau, par exemple. 
que cette initiative est prise deux années avant l'entrée en vigueur de l'ALENA.

Quant au niveau vertical, il convient de souligner que les compromis auxquels ont consenti les gouvernements fédéraux relativement aux prérogatives qui ne relèvent pas de leur compétence et qui appartiennent plutôt aux gouvernements infra-étatiques ont été, dans l'ensemble et jusqu'à un certain point, possibles grâce à la connivence ou, à tout le moins, grâce à une certaine collaboration de la part des gouvernements provinciaux ou étatiques eux-mêmes. Comme quoi le passage au régionalisme économique actuel s'accompagne d'une redéfinition des modes traditionnels de consultation entre ces deux niveaux de gouvernement: que ce soit au Canada ou aux États-Unis, les pouvoirs centraux ont mis en place de nouvelles stratégies de consultation des provinces et des États tout au long des deux rondes successives de négociations des accords de 1989 et de 1994. Aux ÉtatsUnis, l'instance responsable de cette consultation est le United States Trade Representative (USTR) qui nomme, à la demande de la Western Governors Association déjà mentionnée, entre autres, un coordinateur pour les affaires d'État, c'est-à-dire un NAFTA Coor-dinator for State Matters.

Au Canada, le gouvernement fédéral a eu successivement recours à des comités dans lesquels siégeaient des représentants du centre et de chacune des provinces et, à l'inverse, certaines provinces avaient mis sur pied leurs propres comités; on rappellera à ce propos que le gouvernement Bourassa avait créé le comité Warren au moment de la négociation de l'ALE. Au demeurant, il était acquis de part et d'autre, puisque cela fait partie de la pratique constitutionnelle au pays, que les dispositions touchant les domaine de compétence provinciale n'entreraient en vigueur que lorsque les législatures provinciales auraient sanctionné les termes de l'accord. Cette exigence s'applique tout particulièrement aux deux accords parallèles de l'ALENA, et notamment à l'Accord [nord-américain] de coopération dans le domaine du travail (ANACT), qui prévoit un mécanisme particulièrement complexe de sanction et d'application, avec des seuils de représentativité minimaux dans les secteurs couverts, de sorte que les dispositions de l'ANACT ne peuvent entrer en vigueur qu'une fois qu'un nombre déterminé de provinces l'auront sanctionné dans les domaines couverts $^{40}$.

40 «Au Canada, la mise en œuvre effective de l'Accord exige la participation des provinces. Une entente interne régit par conséquent les modalités de collaboration entre les gouvernements fédéral et provinciaux signataires. L'accord intergouvernemental canadien (AIC) prévoit la création d'un comité gouvernemental constitué des ministres responsables du travail ou de leurs délégués, secondé par un comité de hauts fonctionnaires de chaque gouvernement signataire. Ces deux comités fonctionnent 
D'ailleurs, comme il a été noté à la section précédente, certaines dispositions de l'ALENA institutionnalisent la consultation entre les deux ordres de gouvernement, une procédure qui correspond sans doute à l'esprit qui règne dans les régimes fédéraux canadien et américain, mais une pratique «peu habituelle, sinon même fondamentalement inconnue en droit mexicain ${ }^{41}$ », d'où le résultat que certains ont pu voir dans la sanction du recours à des audiences publiques ou hearings à l'annexe 803.3 de l'ALENA, l'introduction d'une procédure relevant de la common law dans le droit interne mexicain. Ajoutons que cette innovation a été reprise par les autorités et intégrée au droit mexicain puisqu'on trouve désormais le recours à des audiences publiques inscrit à l'article 81 de la nouvelle Loi sur le commerce extérieur du 27 juillet $1993^{42}$. L'enjeu, pour le gouvernement mexicain, consiste ainsi à adapter un régime fédéral très centralisé, à l'intérieur duquel l'autorité fédérale dispose d'un nombre important de pouvoirs discrétionnaires pour résoudre les conflits, à un contexte d'ensemble dans lequel les deux autres partenaires pratiquent un fédéralisme de contrepoids (adversarial system) où l'on a davantage recours à la participation des partenaires dans la solution des conflits ${ }^{43}$.

Nous avons ainsi, d'un côté, là où les pouvoirs fédéraux exerçaient leur pleine souveraineté en matière d'affaires extérieures et de commerce international, une implication originale plus forte de la part des gouvernements infra-étatiques dans l'économie mondiale, un processus qui correspond, selon certains auteurs, à rien de moins qu'à une «perforation des souverainetés» étatiques ${ }^{44}$, mais nous avons également, d'un autre côté, un ensemble de phénomènes plus précis et plus ponctuels auxquels correspondent les multiples implications latérales directes qui ont en commun cette caractéristique de se déployer à l'intérieur d'un périmètre à la fois sous-régional et transnational, ou trans-étatique si l'on préfère. En ce sens, et contrairement à ce que soutient la thèse dite de la «globalocalisation», les interventions des provinces et États contribuent moins à globaliser de manière abstraite ou générale l'économie provinciale ou celle de l'État, moins à globaliser un secteur ou une branche, mais davantage à régionaliser, ou encore à

selon le principe du consensus. Les représentants des provinces qui n'ont pas signé l'AIC peuvent néanmoins participer à titre d'observateurs aux réunions de ces comités.» (R. Blouin et A. Giles [dir.], L'intégration économique en Amérique du Nord et les relations industrielles, Sainte-Foy, Presses de l'Université Laval, 1998, p. 188).

41 H. Fix Fierro et A Lopez, «El Tratado de libre comercio de América del Norte y la globalizacion del derecho», dans J. Witker, ouvr. cité, p. 42.

42 Ibid.

43 Ibid., p. 43.

44 I. C. Duchaced, D. Latouche et G. Stevenson (dir.), Perforated Sovereignties and International Relations. Trans-Sovereign Contacts of Subnational Governments, New York, Greenwood Press, 1988. 
continentaliser l'économie, le secteur ou la branche.

D'ailleurs, en Amérique du Nord en tout cas, provinces et États jouissent depuis déjà longtemps d'une importante autonomie en matière internationale qui les conduit à intervenir directement dans l'espace international en adoptant certaines politiques ou en sanctionnant certaines normes qui vont à l'encontre de celles que défendent les gouvernements centraux, comme ce fut le cas lorsque les États de Washington et de l'Oregon ont adopté des politiques reconnaissant les «deux Chines», ou lorsqu'une vingtaine d'États avaient rapatrié leurs fonds de pension de l'Afrique du Sud sous le régime de l'apartheid, ou comme c'est encore le cas quand la province de Québec cherche à se faire octroyer un statut autonome à l'intérieur de la communauté des pays francophones.

Ce n'est donc pas cet aspect qui nous apparaît le plus révélateur des transformations en cours. En effet, il se passe à l'heure actuelle quelque chose de plus déterminant qui reflète peut-être l'émergence d'une nouvelle conscience commune de la part des unités infra-étatiques et de certains gouvernements centraux face au processus d'intégration économique en cours dans les Amériques. Cette «nouvelle alliance» brouille quelque peu les lignes de démarcation dont il a été question jusqu'à maintenant, puisqu'elle lie désormais les pouvoirs législatifs de manière oblique, c'est-à-dire qu'elle conduit au rapprochement entre pouvoirs législatifs centraux et infra-étatiques. De plus, ce n'est sans doute pas par hasard que cette stratégie et ce rapprochement ont été aménagés dans le cadre de l'intégration hémisphérique. Voyons rapidement de quoi il s'agit.

On se souviendra que le processus d'intégration économique à l'échelle de l'hémisphère a été entrepris à l'instigation des États-Unis, à l'occasion du Sommet des Amériques convoqué à Miami en décembre 1994. Ce vaste projet prévoit, entre autres choses, la création d'une Zone de libre-échange des Amériques (ZLEA) d'ici 2005, une zone qui rassemblerait 34 pays: 12 dans la Caraibe, sept en Amérique centrale, 12 en Amérique du Sud et trois en Amérique du Nord. Ce projet réunit tous les pays dont le chef d'État ou de gouvernement a été élu démocratiquement, une exigence qui permet de justifier l'exclusion de Cuba des discussions. À leur tour, ces pays rassemblent au total 164 gouvernements infra-étatiques, qu'il s'agisse de provinces ou d'États, à l'intérieur de six fédérations: 22 au Venezuela en incluant le district fédéral, mais sans compter un territoire et les «dépendances fédérales», 26 au Brésil et 23 en Argentine, auxquels il convient d'ajouter les 93 de l'Amérique du Nord dénombrés plus haut (13 au Canada avec les trois territoires, 50 aux États-Unis et 31 au Mexique). À ce nombre, et pour faire bonne mesure, il faudrait ajouter les cinq parlements supra- 
étatiques que sont le Parlement latino-américain, le Parlement andin, le Parlement centre-américain, l'Assemblée parlementaire de la Communauté de la Caraibe et la Commission parlementaire conjointe du Mercosur.

Ces 164 gouvernements infra-étatiques et ces 35, y compris Cuba, gouvernements centraux, de même que les cinq parlements régionaux, ont été convoqués, en septembre 1997, par l'Assemblée nationale du Québec, à la Première Conférence des parlementaires des Amériques (COPAM). Cette initiative apparaît comme un indice de l'émergence d'une communauté d'intérêts chez les assemblées, parlements et autres instances législatives face à un processus d'intégration hémisphérique défendu par les chefs d'État et les pouvoirs exécutifs centraux.

Le problème qui est très spécifiquement en cause ici, c'est celui du rôle que jouent désormais les pouvoirs exécutifs, non seulement face à leur propre pouvoir législatif, mais face aux autres niveaux de pouvoirs. Les exécutifs des gouvernements centraux, provinciaux ou étatiques s'arrogent de nouvelles prérogatives, ils entreprennent de nouvelles actions, dont les effets sont déterminants, comme nous l'avons vu, non seulement à l'intérieur de leurs propres domaines de compétence, mais également à l'intérieur d'aires qui relèvent de l'autre niveau de pouvoir. Qu'advient-il alors de la question de l'imputabilité et des responsabilités des élus face à leurs propres commettants? Doit-on, au nom de la démocratie, accepter la centralisation des initiatives et renvoyer l'aménagement interne des négociations à des formes plus ou moins occultes de consultation ou de collaboration entre des exécutifs situés à différents niveaux de gouvernement? Ne sommes-nous pas en train, au nom de la démocratie, d'accroître indûment les prérogatives des chefs d'État et de gouvernement, bref, de sanctionner une véritable démocratie d'exécutif?

Ces questions, et plusieurs autres de même nature, ont été au centre des débats de la COPAM qui a terminé ses travaux par l'adoption d'une «Déclaration finale» dans laquelle les participants ont fait état de leurs préoccupations face au processus d'intégration hémisphérique. On peut se demander, par exemple, dans quelle mesure les formes et les modalités des négociations en cours entre chefs d'État et de gouvernement «démocratiquement élus» permettent d'affronter les problèmes liés à l'intégration économique et s'il ne faudrait pas plutôt, par exemple, «recourir davantage à la coopération et au dialogue transfrontaliers ${ }^{45}$ », c'est-à-dire favoriser l'implication latérale directe, au lieu de devoir systématiquement concentrer les négociations dans une structure verticale plus ou moins adaptée et transparente.

45 COPAM, Éléments de réflexion, Québec, Gouvernement du Québec, 1997, p. 27. 
On trouvera une réponse intéressante à cette préoccupation dans le fait que les parlementaires ont décidé de mettre sur pied un réseau des parlementaires des Amériques et que ce Réseau entretient depuis lors des liens de plus en plus serrés avec d'autres composantes des sociétés civiles des Amériques. C'est ainsi qu'au Sommet des peuples des Amériques à Santiago, en avril 1998, un événement organisé par une demi-douzaine de coalitions nationales sur le libre-échange et tenu en marge du Deuxième Sommet des Amériques, les parlementaires se sont réunis dans un forum parlementaire et, à l'instar des huit autres forums parallèles, ils ont dénoncé les formes et les modalités du processus d'intégration en cours. Il s'agit là d'une initiative à la fois intéressante et révélatrice qui illustre le problème de l'imputabilité évoqué par la commission Macdonald et qui renvoie à la question de la légitimité politique de l'intégration économique à grande échelle. Cette question représente en quelque sorte la version panaméricaine du déficit démocratique.

\section{Conclusion}

Dans la foulée des nombreux travaux réalisés ces récentes années autour de la question de l'intégration économique régionale et de ses effets sur les niveaux de gouvernement, nous avons voulu présenter quelques réflexions et ouvrir quelques pistes d'analyse susceptibles de rendre compte des défis et des contraintes auxquels font face les gouvernements infra-étatiques dans le contexte de l'intégration des Amériques. Nous avons voulu faire ressortir que la globalisation rend de plus en plus floue la ligne de partage des pouvoirs entre le gouvernement central et les gouvernements infra-étatiques, notamment celle qui reposait sur la distinction traditionnelle entre le commerce intérieur et le commerce international. Plus le commerce gagne en importance dans la conduite des affaires internationales, plus les gouvernements centraux doivent composer avec d'autres acteurs publics, aussi bien au cours des négociations commerciales elles-mêmes qu'au moment de la mise en œuvre des engagements pris, d'où il résulte que l'on assiste, à travers ce mouvement de va-et-vient, de bas en haut et de haut en bas, à une refonte progressive des institutions dont l'objet n'est pas simplement de favoriser l'intégration des espaces économiques nationaux à l'économie mondiale, ou continentale, pour nous en tenir au champ couvert par notre étude, mais dont l'objet vise la mise en place de nouveaux cadres normatifs qui conduisent à une redéfinition des frontières entre espaces publics nationaux et infranationaux.

Loin d'être des acteurs passifs dans ce processus, les exécutifs des gouvernements infra-étatiques surtout interviennent désormais en leur 
nom et capacité propres de plain-pied sur la scène internationale, initiatives qui posent des difficultés d'un autre ordre. Que doivent alors faire les gouvernements centraux? S'agira-t-il pour eux de laisser faire, d'encadrer ou de brider ces initiatives? Cette question, que nous avons eu l'occasion de soulever à travers notre brève synthèse des conclusions de la commission Macdonald, ne reçoit pas de réponse claire pour le moment. Nous faisons face actuellement à une remise en question profonde des cadres juridiques qui est enclenchée par la mondialisation, ce qu'illustre au mieux la connexion récente et de moins en moins contournable entre intégration économique et révision constitutionnelle, en sorte qu'il reste encore beaucoup à faire pour intégrer tous les éléments pertinents dans un cadre d'analyse global et surtout pour lier les pratiques d'acteurs aux trois niveaux concurremment, central, infraétatique et local.

En attendant, nous avons voulu montrer que l'intégration économique régionale, qui a tant contribué à rapprocher les gouvernements centraux dans les Amériques et qui a favorisé, dans une grande mesure, un plus grand rayonnement du modèle américain de gestion publique, a eu et aura de plus en plus des retombées significatives sur les autres niveaux de gouvernement. Il est intéressant de souligner à ce propos, pour finir, tout l'attrait qu'exerce le modèle américain d'organisation du territoire sur les formes et modalités d'organisation du territoire au Québec, par exemple. C'est ainsi que le Secrétariat au développement des régions a produit et publié une courte et fort intéressante étude consacrée aux avantages du «modèle américain» plus souple et plus diversifié, là où «les approches européennes (France, Allemagne et Suède notamment) abusent peutêtre d'un modèle unique plaqué à tout leur territoire national ${ }^{46}{ }_{\text {». }}$. L'auteur de cette étude souligne de plus la comparabilité dans la taille des deux instances régionales et sous-régionales de développement que représentent d'un côté les 475 conseils régionaux (CRG, avec 547000 habitants, en moyenne) et les 3040 comtés (avec 85000 habitants) aux États-Unis, et de l'autre les 16 conseils régionaux (CRD, avec 475000 habitants) et les 95 municipalités régionales de comté (MRC, avec 74000 habitants) au Québec. Cependant, la comparaison s'arrête là, car les comtés ont bel et bien une existence historique et sociale forte aux États-Unis, tandis que les MRC, en particulier, sont de création récente et très hétérogènes quant à leur mode de fonctionnement. En ce sens, et dans le prolongement de ce que nous avons vu ci-dessus, le comté constitue bel et bien un acteur politique d'un certain poids aux ÉtatsUnis, tandis que la MRC est encore loin d'avoir gagné un statut comparable au Québec où le pouvoir municipal demeure le troisième

46 L.-M. Bouchard, Politiques régionales et territoriales, cahier no 1, Les États-Unis, Québec, Secrétariat au développernent des régions, 1996, p. 17. 
ordre de gouvernement. Mais, quoi qu'il en soit de ces différences, le plus intéressant, et le plus révélateur à la fois, c'est sans doute que des instances gouvernementales provinciales soumettent à la réflexion ce genre d'études et d'analyses comparées, comme quoi les effets de l'intégration économique régionale sollicitent désormais chacun des niveaux et des ordres de pouvoirs ${ }^{47}$.

\section{Dorval BRUNELLE, \\ Département de sociologie \\ Yves BÉLANGER \\ Christian DEBLOCK \\ Département de sciences politiques, \\ Groupe de recherche sur l'intégration continentale Université du Québec à Montréal}

\section{Résumé}

Par le passé, les accords de libre-échange et autres ententes commerciales engageaient au premier chef leurs signataires; ils n'avaient pas d'effets directs sur les autres niveaux de gouvernement. Or cette étanchéité entre les niveaux de gouvernement ne prévaut plus depuis l'entrée en vigueur, le ler janvier 1989, de l'Accord de libreéchange entre le Canada et les États-Unis. En effet, pour la première fois, un accord commercial stipule expressément que ses dispositions devraient s'appliquer également aux États et aux provinces. La question surgit alors de savoir en quoi et comment l'ALE, puis l'Accord de libre-échange nord-américain, en 1994, sont venus modifier les relations et interrelations entre les pouvoirs centraux et les gouvernements infraétatiques. L'article se propose d'aborder ces questions dans une perspective d'ensemble tenant compte des trois Amériques. L'analyse est partagée en trois temps. Dans un premier temps, les auteurs présentent quelques données de base sur les pays et les gouvernements infra-étatiques. Dans un deuxième temps, ils examinent les dispositions pertinentes de l'ALE et de l'ALENA, après quoi ils s'intéressent aux pouvoirs provinciaux ou étatiques en tant qu'acteurs politiques. Cette approche leur permet de mettre en lumière quelques nouveaux aspects de la démocratie d'exécutifs qui semble prédominer dans la mise en place des cadres normatifs propres à la mondialisation économique dans sa phase actuelle.

47 Une récente étude permet de mettre en lumière le lien serré qui existe entre la politique économique libre-échangiste de l'actuel gouvernement péquiste et sa politique régionale (voir Mouvement d'éducation populaire et d'action communautaire du Québec [MEPACQ], La localisation, la régionalisation et... la mondialisation. Enjeux et impacts de la "réorganisation» en cours, Québec, décembre 1997). 
Mots-clés: libre-échange, Accord de libre-échange, Accord de libreéchange nord-américain, gouvernements centraux, provinces, États, gouvernements infra-étatiques, acteurs politiques, démocratie d'exécutifs.

\section{Summary}

In the past, free trade agreements and other trade agreements committed for the most part only their signatories. They had no direct effects on other levels of government. However, this clear line between levels of government ceased prevailing with the implementation of the Free Trade Agreement between Canada and the United States on January 1, 1989. Indeed, for the first time a trade agreement expressly stipulated that its clauses applied equally to the States and the provinces. The question thus emerged as to how and in what ways the Free Trade Agreement and, subsequently, NAFTA (1994) have modified relations and interrelations between central governments and infra-State governments. This article will discuss these questions from an overarching perspective encompassing the three Americas. There are three parts to the analysis. In the first part, the authors present a few basic data about the countries and the infra-State governments. Secondly, they examine the relevant clauses of the Free Trade Agreement and NAFTA. This is followed by a discussion of provincial or State governments as political actors. This approach enables them to cast light on some new aspects of the democracy of executives that appears to be predominating in the implementation of normative frameworks specific to the current state of economic globalization.

Key-words: free trade, Free Trade Agreement, North American Free Trade Agreement, central governments, States, infra-State governments, political actors, democracy of executives.

\section{Resumen}

En el pasado, los acuerdos de libre comercio y otros acuerdos comerciales comprometían en primer lugar a las personas que firmaban, razón por la cual dichos acuerdos no tenían efectos directos sobre los otros niveles del gobierno. Ahora bien, este hermetismo entre los niveles de gobierno dejó de prevalecer a partir del 10. de enero de 1989, con la puesta en vigencia del Tratado de libre-comercio entre Canadá y los Estados Unidos (ALE). En efecto, por primera vez un acuerdo comercial estipula expresamente que sus disposiciones deberán aplicarse igualmente tanto en los Estados como en las provincias. Surge entonces la cuestión de saber en qué y cómo el Tratado de libre- 
comercio norteamericano (ALENA) vino, en 1994, a modificar las relaciones e interrelaciones entre los poderes centrales y los gobiernos infra-estatales. Estas preguntas son abordadas por los autores dentro de una perspectiva de conjunto que concierne a las tres Américas. El análisis es presentado en tres partes. En la primera, los autores presentan algunos datos de base sobre los países y los gobiernos infra-estatales. En la segunda, ellos examinan las disposiciones pertinentes del Tratado de libre-comercio (ALE) y del Tratado de libre-comercio norteamericano (ALENA) que serán estudiadas. Por último, los autores se interesan en los poderes provinciales o estatales en tanto que actores políticos. Este enfoque les permite sacar a la luz algunos nuevos aspectos de la democracia de ejecutores que parece predominar en la puesta en práctica de cuadros normativos propios a la mundialisación económica dentro de su fase actual.

Palabras claves: libre-comercio, Tratado de libre-comercio, Tratado de libre-comercio norteamericano, gobiernos centrales, provincias, Estados, gobiernos infra-estatales, actores políticos, democracia de ejecutores. 